\title{
Mögliche Wege der Übertragung viraler Erkrankungen beim gemeinsamen Zubereiten von Drogen zum i.v. Konsum - Konsequenzen für die Prävention*
}

\author{
Daniel Meili ${ }^{1}$ \\ Juraj Marcinko ${ }^{1}$ \\ Barbara Bertisch-Möllenhoff ${ }^{1}$ \\ Milo Huber ${ }^{1}$ \\ Felix Davatz ${ }^{1}$ \\ Jen Wang ${ }^{2}$
}

\author{
Possible Transmission Routes of Viral Diseases During Communal Preparation of \\ Drugs for i. v. Use
}

\section{Zusammenfassung}

Ziel: Die Studie beschreibt detailliert, welche Techniken beim Zubereiten von Drogen zum intravenösen Konsum in Gruppen angewandt werden (indirect sharing) und wie das Infektionsrisiko eingeschätzt wird. Illustriert wird, wie kleinste Blutmengen und dadurch auch Viren über mehrfach verwendete Utensilien und Flüssigkeiten weitergegeben werden können.

Methode: Befragung von 72 Patienten ${ }^{1}$ in einem Methadonprogramm, die zum Zeitpunkt der Untersuchung intravenösen Beikonsum hatten.

Ergebnis: Nur 19\% der i.v. Konsumierenden geben an, nie Drogen in Gruppen zuzubereiten. Durch die Techniken des Aufteilens (Frontloading, Backloading, Ausgleich über den Löffel etc.) gelangt die Drogenlösung häufig mit fremden, gebrauchten Spritzen, Löffeln oder Filtern in Kontakt. Die Mehrheit erachtet das Tauschen von Spritzen und Nadeln als Infektrisiko, hingegen stufen die meisten das gemeinsame Zubereiten von Drogen als harmlos ein. Schlussfolgerungen: Drogenlösungen beim Drogenteilen müssen potenziell als virenkontaminiert betrachtet werden. Das Hepatitis-C-Infektionsrisiko steht dabei im Vordergrund. Neue präzise Präventionsbemühungen sind notwendig: bei jeder Injektion - ob alleine oder in Gruppen - Verwenden von neuen Nadeln und Spritzen, als Lösungsmittel NaCl-Wasser aus Einmalgebinden, Filter nur einmal verwenden und die restlichen Utensilien (Löffel, Wasserbehälter) adäquat reinigen.

\section{Schlüisselwörter}

Drogen · Infektionsrisiko • indirect sharing • intravenöser Konsum • Hepatitis C · Prävention

1 Zur Verwendung der männlichen Pluralform: Gemeint sind hier wie im Folgenden Patientinnen und Patienten.

\section{Abstract}

Goal: The study describes in detail the techniques used for the group preparation and subsequent intravenous drug use. Special consideration is given to „indirect sharing“ practices and to how the users judge the infectious risk associated with those practices. It shows how minimal amounts of blood and thereby also viral particles can be transmitted by the repeated use of drug preparation equipment and liquids.

Study Design: Interrogation of 72 patients participating in a methadone substitution programme who continued i.v. drug use at the time of the investigation.

Result: Only $19 \%$ of the i.v. drug users reported never preparing drugs in groups. Via drug sharing techniques (frontloading, backloading, flushing of the syringes etc.) the drug solution frequently comes into contact with used syringes, spoons and filters of other drug users. The majority of users consider the exchange of syringes and needles as a risk for infection. On the contrary, most of the study participants judged the common preparation of drugs to be harmless.

Conclusions: With communal preparation and sharing, drug solutions must be regarded as potentially virus contaminated. In this context, the risk for hepatitis $C$ is of utmost concern. New targeted prevention efforts are necessary: for every injection, whether alone or in a group, use new needles and syringes, disposable $\mathrm{NaCl}$ solution ampoules, single-use filters and adequate cleaning of all other utensils (spoon/cooker).

\section{Key words}

Intravenous Drug Use · Indirect Sharing · Infectious Risk · Hepatitis $\mathrm{C} \cdot$ Prevention

Anmerkung

* Der ausführliche Bericht ist unter www.arud-zh.ch zu finden: Marcinko J. Intravenöser Drogenkonsum: Genau betrachtete Praktiken des Injektionsverhaltens. Eine detaillierte Befragung von MethadonbezügerInnen. 1999.

Institutsangaben

${ }^{1}$ ARUD Zürich, Arbeitsgemeinschaft für risikoarmen Umgang mit Drogen

2 Institut für Sozial- und Präventivmedizin, Universität Zürich

Korrespondenzadresse

Dr. med. Daniel Meili · ARUD Zürich · Konradstrasse $1 \cdot 8005$ Zürich $\cdot$ Schweiz

Bibliografie

Suchttherapie Supplement 2002; 3: S20-S26 @ Georg Thieme Verlag Stuttgart · New York · ISSN 1439-9903 
Die HIV-Epidemie der 80er Jahre führte rasch zu intensiven Forschungsbemühungen. Es zeigte sich, dass neben Bluttransfusionen und sexuellen Kontakten der Tausch von gebrauchten Spritzen und Nadeln („direct sharing“) unter i.v. Drogenabhängigen zu den bedeutendsten Übertragungsrisiken des HI-Virus gehört [1]. Breit angelegte Präventionskampagnen in der Schweiz („STOP-AIDS“) mit der Hauptbotschaft „sexuelle Kontakte mit Präservativen“ und „kein Nadel- und Spritzentausch beim intravenösen Drogenkonsum“ zeigten Erfolg. Mit dem Aufbau von Angeboten zum Tauschen von Spritzen hat dies bewirkt, dass Spritzen und Nadeln nur noch sehr selten getauscht werden [2]. Die HIV-Inzidenz reduzierte sich bei Personen, die illegal „harte“ Drogen konsumieren, und die -Prävalenz sank je nach Region unterschiedlich stark von 45-53\% im Jahre 1989 auf unter 15\% im Jahre 1998 [3].

Das erst Ende der 80er Jahre identifizierte Hepatitis-C-Virus (HCV), erkannt als Verursacher der hauptsächlich blutübertragenen Leberentzündung [1], verbreitete sich vor allem unter Drogenabhängigen in der Folgezeit hingegen sehr rasch $[1,3,4]$. In der Poliklinik Zokl1 der ARUD Zürich (Arbeitsgemeinschaft für risikoarmen Umgang mit Drogen), einem der größten Methadonprogramme der Schweiz mit ca. 500 Behandlungsplätzen, waren bereits 1996 über 60\% der Methadonbezieher HCV-positiv [5]. In der Schweiz waren es bei denjenigen, die zwischen 1994 und 1996 neu in die heroingestützten Behandlungen eingetreten sind, über $80 \%$ [3]. Über generelle Zahlen in der Drogenszene liegen keine gesicherten Angaben vor. Aus den Seroprävalenz-Untersuchungen in den heroingestützten Behandlungen hat sich gezeigt, dass bei einer Abhängigkeitsdauer von 5 bis 9 Jahren 76\%, bei einer Abhängigkeitsdauer von 10 bis 15 Jahren 90\% der Patienten Hepatitis-C-positiv sind [3].

Bereits Anfang der 90er Jahre wurde in Bezug auf die Übertragung von HIV unter Drogenabhängigen auf einen weiteren Übertragungsmechanismus hingewiesen: das „indirect sharing“. Dies sind nach Koester Praktiken, bei welchen Konsumenten ohne Spritzentausch Injektionsmaterialien gemeinsam benutzen oder Drogen nach dem Zubereitungsprozess teilen (Tab. 1) [6]. 1990 berichtete ebenfalls Koester aus Colorado über das Ansteckungsrisiko mit HIV bei gemeinsamer Verwendung von Wassergefäßen, Löffeln und Filtern. Er vermutete, dass auf diese Weise eine Infektion übertragen werden kann, auch wenn zuletzt sterile Spritzen verwendet werden [7]. Grund berichtete zur gleichen Zeit über die Techniken des Frontloadings (Tab.1) in den Niederlanden. Er vermutete, dass diese Technik für einen beträchtlichen Teil der Neuinfektionen mit dem HI-Virus verantwortlich ist [8]. 1991 berichtete Loimer aus Wien über das Löffelteilen als einen wesentlichen Risikofaktor bei der Ausbreitung der HIV-Infektion unter Drogenkonsumenten, die niemals gebrauchte Spritzen oder Nadeln verwendet haben [9]. Er lenkte erneut das Augenmerk auf die gemeinsame Verwendung von Spülwassergefäßen und Filtern. 1993 fand Jose in New York eine signifikante positive Korrelation zwischen Backloading (Tab.1) und HIV-Positivität [10].

Bezüglich der Hepatitis-C-Übertragung liegen folgende Erkenntnisse vor: 1995 zeigte Stark aus Berlin, dass Frontloading ein unabhängiger Risikofaktor für HCV-Infektionen ist [11]. 2000 wies
Injizierbare Lösung von Heroin, Kokain und Cocktail (Gemisch von Heroin und Kokain) Pulverförmiges Heroin wird zum Auflösen in einen feuerfesten Behälter (meist einen Löffel) gegeben, zugefügt werden Wasser und Ansäuerungsmittel. Das Gemisch wird über einer Flamme erhitzt. Falls nötig, werden zusätzliches Wasser und Ansäuerungsmittel beigemengt.

Kokain ist in kaltem Wasser ohne Ansäuern löslich; meistens wird es auf diese Weise zubereitet.

Für das Auflösen von Cocktails wird Heroin mit der obigen Technik vorbereitet. Oft wird dann zum Abkühlen aus einer Spritze etwas kaltes Wasser beigegeben. Anschließend wird das Kokainpulver zugefügt und mit einem Werkzeug, meistens einem Bestandteil der Spritze, umgerührt. Falls die Lösung zu dickflüssig ist, wird nochmals Wasser zugegeben. Anschließend wird ein Filter (kleines Kügelchen zumeist aus Zigarettenfilter) in der Lösung platziert und die Drogenlösung wird ohne Nadel durch diesen Filter aufgezogen. Nach dem Aufsetzen der Nadel wird zur Kontrolle, ob die Nadel gut in der Vene platziert ist, vor der Injektion eine kleine Blutmenge in die Spritze gezogen.

Techniken des Drogenteilens

Simultanes Aufziehen: Zwei Spritzen werden simultan ohne Nadel auf den Filter gepresst und die vorbereitete Lösung wird gleichzeitig von einer Person aufgezogen. Bei ungleichen Mengen wird der Überschuss in den Löffel zurückgegeben und mit der anderen Spritze aufgezogen.

Frontloading: Dabei wird die gesamte Drogenmenge in eine Spritze aufgezogen. Mit einer Nadel bestückt, wird die Hälfte durch die Frontöffnung in die Empfängerspritze umgefüllt.

Backloading: Es wird aus der Empfängerspritze der Kolben herausgezogen und aus der Geberspritze wird mit oder ohne Nadel die Drogenlösung von hinten eingespritzt.

Rest überlassen: Mehrere Konsumenten ziehen ihren Drogenanteil nacheinander über den Filter in ihre Spritze auf. Ausgleich nach obigen Techniken. Kontamination mit Blut

Damit es zu einer Kontamination der Drogenlösung und der Utensilien kommen kann, muss Blut in die Spritze gelangen: Sobald die Nadel in der Vene platziert ist, wird eine kleine Menge Blut in die Spritze gezogen, um die korrekte Lage zu prüfen. Nach Injektion der gesamten Drogenlösung bleibt immer eine kleine Blutmenge in Nadel und Spritze $[18,19]$.

Thorpe bei intravenös Drogenkonsumierenden in Chicago ein signifikant erhöhtes Risiko bei gemeinsamer Benutzung von $\mathrm{Zu}$ bereitungsgefäß, Spülwasser und Filtern nach [12]. Ebenfalls 2000 konnte Denis bei belgischen Heroinkonsumenten neben Spritzentausch den Tausch von Filtern, aber nicht die gemeinsame Benutzung von Löffeln als ein statistisch signifikantes HCV-Risiko erfassen [13]. 2001 fand Hagan bei intravenös konsumierenden Drogenpatienten in Seattle eine dreifach erhöhte Hepatitis-C-Serokonversionsrate bei Spritzentausch, Filtertausch und gemeinsamer Benutzung des Gefäßes zur Drogenzubereitung [14].

US-amerikanische Richtlinien enthielten bereits seit 1996 bzw. 1997 den Ratschlag für Ärzte, ihre drogenabhängigen Patienten darauf hinzuweisen, auch anderes Injektionsmaterial als Spritzen nicht mit anderen Konsumenten zu tauschen $[15,16]$.

Um konkrete Präventionsbotschaften $\mathrm{zu}$ formulieren, interessierten uns die detaillierten Praktiken und Begleitumstände des intravenösen Drogenkonsums unter den gegebenen soziokulturellen Verhältnissen in Zürich. Die möglichen Wege, wie kleinste Blutmengen und dabei auch Viren beim ,indirect sharing“ weitergegeben werden können, stehen im Folgenden im Vordergrund. Obwohl die Daten nicht mehr ganz neu sind, haben die daraus gewonnenen Erkenntnisse nicht an Aktualität verloren. 


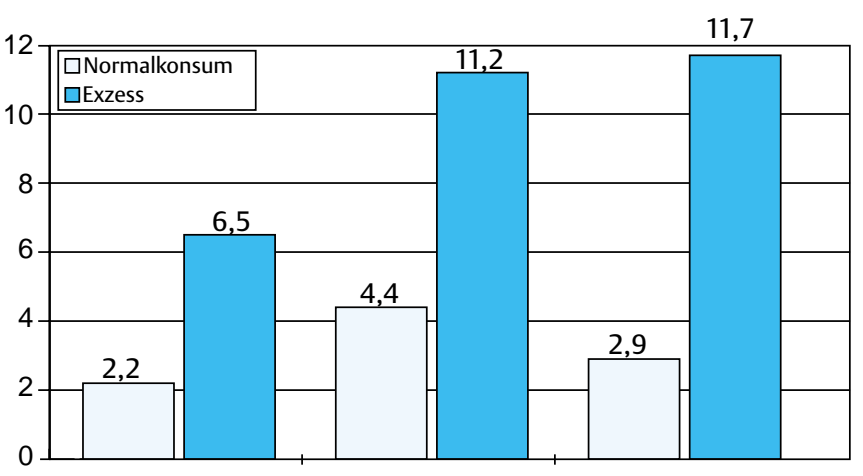

Untersucht wurden Patienten aus der Poliklinik für methadongestützte Behandlung, Zokl1 der ARUD Zürich (Arbeitsgemeinschaft für risikoarmen Umgang mit Drogen), die während der Teilnahme am Methadonprogramm bei der dreimonatlichen Befragung zur Basisevaluation angegeben hatten, im Laufe der vergangenen Wochen intravenös Drogen konsumiert zu haben. Es wurden also nur solche Patienten in die Untersuchung eingeschlossen, die neben der methadongestützten Behandlung Beikonsum hatten. Als Basis für die Rekrutierung dienten 350 Personen (Stichtagerhebung aller Patienten im Methadonprogramm im Dezember 1996). Zwei Drittel gaben keinen intravenösen Beikonsum an. 117 entsprachen den Einschlusskriterien, 68 (58\%) davon nahmen an der Studie teil. Zusätzlich wurden vier neu eintretende Männer nachträglich in die Studie einbezogen. Erfragt wurden das exakte technische Vorgehen beim Konsum, das Hygieneverhalten und die Beurteilung des Infektionsrisikos, welches an die einzelnen Schritte gekoppelt ist.

Die Interviews wurden als persönliche Befragung mittels 24-seitigem Fragebogen (mit überwiegend geschlossenen Fragen) durchgeführt. Den Fragebogen entwickelten wir anhand von Erfahrungen aus der täglichen Arbeit in der Poliklinik Zokl1 in Zusammenarbeit mit dem Institut für Sozial- und Präventivmedizin der Universität Zürich. Die Interviewteilnehmer erhielten Sfr. 20.- Entschädigung.

Die Daten wurden in einer Exceltabelle eingegeben und mittels SPSS statistisch ausgewertet.

Ergebnisse

Wir berichten hier lediglich diejenigen Resultate, die im Zusammenhang mit der möglichen Weitergabe viraler Erkrankungen stehen. Zusätzlich erhobene Informationen zur Gefährdung durch die Verwendung problematischer Ansäuerungsmittel, bakterielle Kontaminationsmöglichkeiten u. a. werden nicht referiert.

Merkmale der Stichprobe: Der Frauenanteil beträgt ein Viertel. Die Befragten sind im Durchschnitt 30 Jahre alt und konsumieren durchschnittlich seit 9,1 Jahren Heroin, davon seit 8 Jahren in intravenöser Form. Durchschnittlich seit 7,3 Jahren konsumieren sie zusätzlich Kokain (seit 4,8 Jahren intravenös), seit über 5 Jahren auch in Form von Cocktails (Gemisch von Heroin und Kokain).

\section{Aktueller Beikonsum}

Zwei Drittel konsumieren neben der Substitution in verschiedenen Mengen i.v. Heroin (77\%), die Hälfte Kokain (47\%) und 82\% in gemischter Form (Cocktails).

An einem durchschnittlichen Tag wird Heroin im Mittel 2,2-mal, Kokain und Cocktails werden 4,1-mal bzw. 2,9-mal gespritzt.

Als exzessiver Konsum wurde ein Konsumverhalten definiert, welches sich deutlich vom oben beschriebenen normalen Konsumverhalten unterscheidet. Es ist ein von den Interviewteilnehmern selbst definiertes Verhalten.
Abb. 1: Durchschnittliche Anzahl Injektionen pro Tag bei normalem und exzessivem Konsum $(n=72)$.

Phasen exzessiven Konsums gaben $31 \%$ bei Heroinkonsum an, $39 \%$ bei Kokainkonsum und $76 \%$ bei Cocktailkonsum.

In Abb. 1 wird sichtbar, dass sich die Anzahl der Injektionen beim „normalen“ Konsum deutlich von dem als exzessiv empfundenen Konsum unterscheidet.

\section{Soziale Umgebung beim Drogenkonsum}

Erfragt wurden Anwesenheit anderer Personen beim Drogenkonsum, der Ort, die Kooperation beim Zubereiten und Konsumieren und das Teilen von Utensilien.

Die meisten bevorzugen zwar alleine zu konsumieren (56\%). Eine Minderheit fühlt sich in Anwesenheit anderer Konsumenten sicherer. Sie schätzt dabei auch die Möglichkeit für Gespräche. Allerdings konsumieren nur $15 \%$ immer allein. Am häufigsten wird in Gegenwart anderer Abhängiger konsumiert (,immer/meistens“ in $15 \%$, „manchmal/selten“ in 56\%). Meist ist nur eine andere Person (57\%), zwei in $26 \%$, mehr als zwei Personen in $16 \%$ anwesend.

Am häufigsten (,immer/meistens“ in 81\%) wird in der eigenen Wohnung konsumiert. An zweiter Stelle stehen die Einrichtungen der „Kontakt- und Anlaufstellen“2. Danach folgt der Konsum bei Freunden und Bekannten. Regelmäßiger Konsum auf der Gasse wird noch von $10 \%$ der Befragten angegeben.

Das Gruppenverhalten spiegelt sich auch in der Art der Drogenbeschaffung wider: Die Drogenbeschaffung geschieht häufig in Kleingruppen. Dabei sind unterschiedliche Grade der Kooperation zu beobachten. Manche der folgenden Tätigkeiten werden von Einzelnen für die ganze Gruppe ausgeführt: Geldbeschaffung, Suche von Dealern und Drogenkauf.

Die Spritzen packen drei Viertel der Befragten selber aus. Das Auflösen der Drogen und Aufziehen der Spritzen überlässt knapp die Hälfte der Befragten oft bis immer anderen Drogenkonsumenten.

\footnotetext{
2 „Kontakt- und Anlaufstellen“: Gassenzimmer der Stadt Zürich, in denen Drogen unter Aufsicht konsumiert werden können.
} 
Die mit der Stoffinjektion verbundenen Tätigkeiten wie Venensuche, Reinigung der Injektionsstelle, Injizieren der Drogen, Reinigen und Versorgen gebrauchter Spritzen werden von einer groBen Mehrheit der Befragten (90\%) selbst durchgeführt.

\section{Umgang mit Spritzenutensilien}

Zum besseren Verständnis sind die Methoden der Zubereitung von Heroin und Kokain zum intravenösen Konsum und die Techniken des Drogenteilens in Tab. 1 dargestellt.

Die wichtigsten Utensilien, bei denen eine Kontamination durch Viren möglich ist, sind Löffel, Rührwerkzeug, Wasser, Schöpfgefäß, Filter, Spritzen und Nadeln:

Löffel: In über $80 \%$ der Fälle werden zum Auflösen der Drogen Tee- oder Esslöffel verwendet. Einige behelfen sich mit Teilen von Aludosen. 36\% benutzen den Löffel immer oder meistens gemeinsam mit anderen, nur $18 \%$ verwenden ihn immer alleine. Knapp die Hälfte der Befragten hat in den vergangenen 12 Monaten gebrauchte Löffel weitergegeben.

Nur etwa die Hälfte der Konsumenten reinigt den Löffel regelmäßig vor dem Gebrauch.

Der gemeinsame Löffelgebrauch wird von der großen Mehrheit (70\%) als harmlos für eine Infektion angesehen. Das Infektionsrisiko beim gemeinsamen Verwenden von Löffeln wird als wesentlich geringer betrachtet als beim Verwenden fremder, gebrauchter Löffel.

Rührwerkzeug: Mehrheitlich werden Bestandteile der Spritze und deren Verpackung verwendet. Etwa die Hälfte braucht den Spritzenkolben (Kolbengummi). Nur jeder Zehnte reinigt diesen vor dem Gebrauch regelmäßig. Eine regelmäßige Weitergabe oder Annahme findet nicht statt und wird von etwa der Hälfte als sehr gefährlich bezüglich Infektionsrisiko angesehen.

Wasser und Schöpfgefäß: Die überwiegende Mehrheit der Befragten verwendet kaltes Leitungswasser (71\%) zum Auflösen der Drogen. Knapp ein Viertel benutzt regelmäßig steriles $\mathrm{NaCl}-$ Wasser (24\%). Von 10\% wird warmes Leitungswasser gebraucht. Gelegentlich wird aber auch Wasser aus öffentlichen Brunnen, Wasserlachen und Toiletten verwendet (Mehrfachnennung). Teilweise wird fließendes Wasser aufgezogen; $11 \%$ haben das Wasser zuerst in ihre Handfläche oder in den Mund genommen und dann in eine Spritze gefüllt.
Alle Befragten gaben an, das Wasser mit einer Spritze für den Löffel abzumessen. Damit wird die Menge der fertigen Lösung in etwa bestimmt.

Das Schöpfen aus einem Gefäß erleichtert die genaue Abmessung der gewünschten Wassermenge; dies haben $72 \%$ der Befragten im vergangenem Jahr getan. $61 \%$ von ihnen haben das Gefäß mit anderen Konsumenten geteilt. Manchmal schöpft auch einer für andere das Wasser.

Die Einschätzung der Gefährlichkeit des Schöpfens aus einem gemeinsamen Gefäß gibt Abb. 2 wieder.

Nur die Hälfte der Befragten wechselt das Wasser nach jeder Injektion, viele behalten es bis zu einem Tag im Zimmer (41\%). Einige behalten es bis zu einer Woche (9\%).

57 Personen wurden zudem über ihr Wissen bezüglich der $\mathrm{NaCl}-$ Flüssigkeit in Einmalgebinden befragt, deren Verwendung in der Schweiz empfohlen wird. $70 \%$ wissen, wozu die $\mathrm{NaCl}$-Lösung verwendet werden kann. 19\% denken, diese Lösung könne als Gegenmittel bei einer Überdosierung mit Heroin oder Kokain gebraucht werden. $11 \%$ gehen davon aus, diese Lösung würde die Wirkung der gelösten Drogen abschwächen. Insgesamt sind $30 \%$ der Befragten der Meinung, NaCl-Lösung sei ungeeignet zum Auflösen der Drogen. Aus diesem Grund verwenden sie das $\mathrm{NaCl}$-Wasser nicht.

Filter: Fast alle Befragten (99\%) verwenden zum Aufziehen der Drogen immer einen Filter. Eine überwiegende Mehrheit der Befragten verwendet Zigarettenfilter von ungerauchten Zigaretten (93\%), 18\% von gerauchten Zigaretten, $17 \%$ verwenden Watte. Nur 6\% haben Spezialfilter aus der Apotheke verwendet (Mehrfachnennung möglich).

Die Zigarettenfilter werden in der Regel mit den Fingernägeln oder mit den Zähnen herausgezogen, verkleinert, manchmal zwischen den Fingern gerollt und zum Schluss in der Lösung platziert. Die Öffnung einer Spritze wird auf den Filter gesetzt und die Drogenlösung in die Spritze aufgezogen. Eine Spritzennadel wird dabei nicht verwendet. Fast alle legen den Filter nach dem Erhitzen in den Löffel, 3\% vor dem Erhitzen, 1\% manchmal vorher, manchmal nachher.

Nur 39\% der Befragten geben an, ihren Filter nie zu teilen. 61\% haben ihn schon gemeinsam benutzt; 19\% praktizieren dies meistens oder immer. Rund $45 \%$ schätzen dieses Verhalten als gefährlich in Bezug auf HIV- und andere Infektionen ein, während $40 \%$ der Befragten es als harmlos beurteilen. Zudem wird

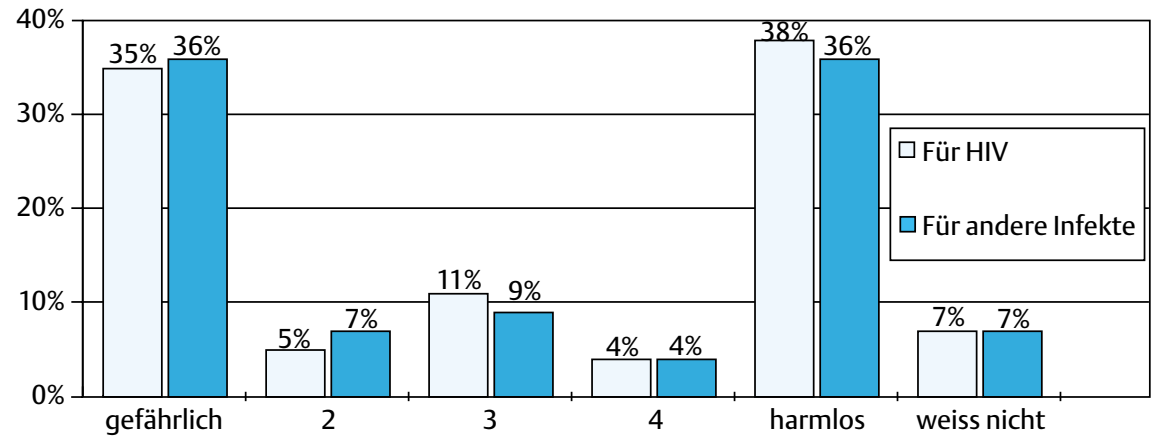

Abb. 2: Einschätzung der Gefährlichkeit des Schöpfens aus einem gemeinsamen Gefäß $(n=55)$. 


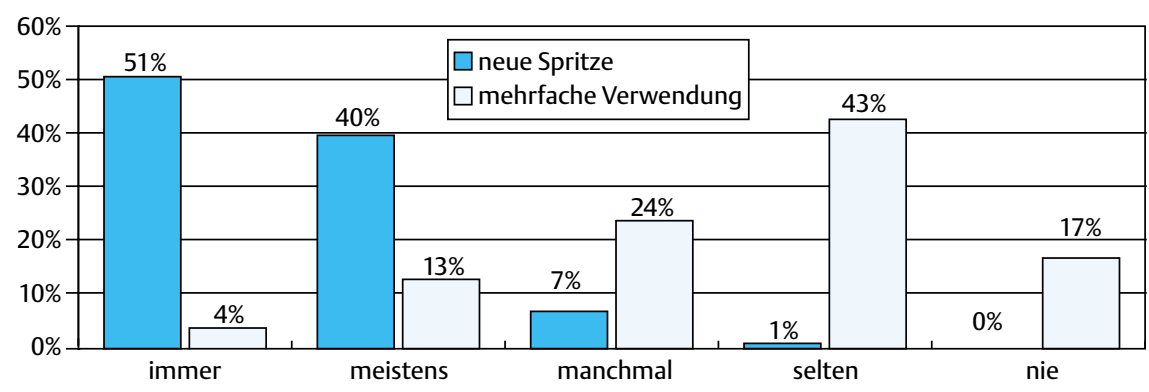

Abb. 3: Mehrfachverwendung der eigenen Spritze $(n=72)$.

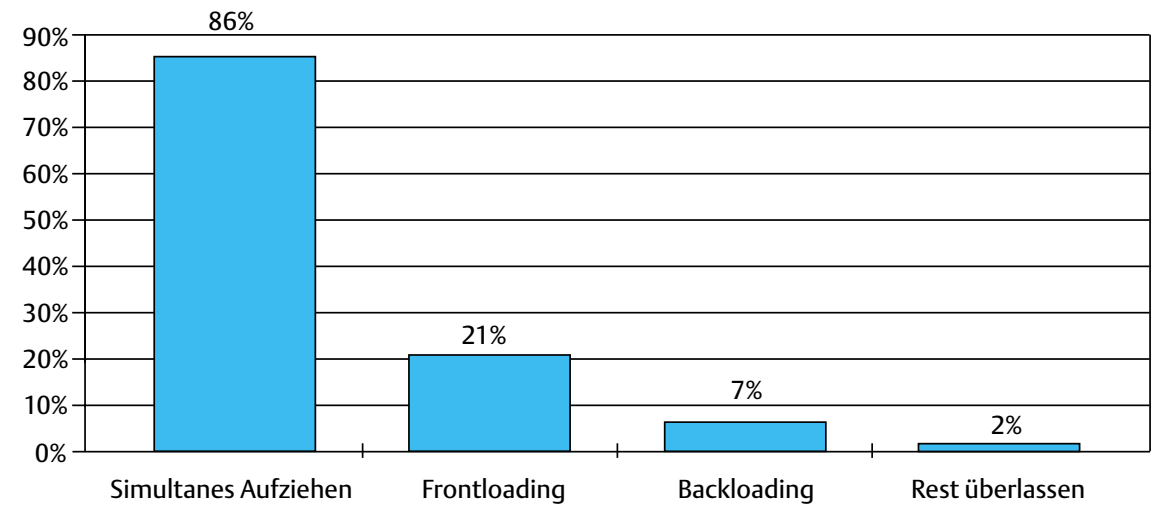

Abb. 4: Techniken des Drogenteilens $(n=58)$.

der gemeinsame Filtergebrauch als weniger gefährlich eingestuft als die Filterannahme, dies vor allem bezüglich HIV.

Die Hälfte verwenden ihre Filter mehrmals (2- bis 15-mal). 20\% bewahren sie zwischen 2 Tagen und einem Monat auf, zumeist feucht verschlossen in einer kleinen Plastiktüte oder einem anderen Behälter. Gebrauchte Filter werden in der Regel nicht aus Bequemlichkeit (17\%), sondern als Stoffreserve (83\%) zum Sammeln und Auskochen aufbewahrt, gelegentlich als Zahlungsmittel für kleinere Gefälligkeiten verwendet oder von in Not geratenen Konsumenten erbettelt.

In Abb. 3 sind die Antworten auf folgende Fragen zusammengefasst: Wie häufig werden neue sterile Spritzen verwendet? Werden Spritzen mehrmals benutzt?

$57 \%$ der Konsumenten geben an, ihre Spritzen vor, $72 \%$, ihre Spritzen nach dem Gebrauch zu reinigen. Nadeln werden weniger häufig gereinigt als Spritzen (vor Gebrauch von $40 \%$ der Konsumenten, nach Gebrauch von $51 \%$ ), sie werden allerdings auch weniger häufig mehrmals verwendet.

Eine überwiegende Mehrheit braucht zur Reinigung eigener gebrauchter Spritzen Wasser. Spritzen und Nadeln werden dabei mehrmals durchgespült. Nur Vereinzelte kochen ihre Spritzen und Nadeln im Wasser oder reinigen sie mit Alkohol.

Das Wasser zur Reinigung ihrer Spritzen und Nadeln entnimmt etwa die Hälfte (fast) immer direkt vom Wasserhahn. Ein Drittel der Befragten entnimmt das Wasser zur Reinigung einem Becher und $14 \%$ entsorgen das Spülwasser wieder in den gleichen Becher.

Fremde Spritzen: Nur zwei der Betroffenen haben innerhalb des letzten Jahres einen Spritzen-/Nadeltausch vorgenommen.
Gut drei Viertel der Befragten (77\%) hält den Gebrauch fremder gebrauchter Spritzen für gefährlich. Obwohl Frauen früher vermehrt Spritzen tauschten, erachten sie dies häufiger für gefährlich als Männer.

\section{Gemeinsames Zubereiten und Konsumieren von Drogen}

Für 20\% der Befragten ist gemeinsames Auflösen die normale Konsumform. $62 \%$ praktizieren dies manchmal bis selten. Nur $19 \%$ geben an, nie gemeinsam Drogen zuzubereiten. Etwa die Hälfte konsumiert in konstanten Konsumgruppen. Drei Viertel der Betroffenen konsumieren beim Teilen (fast) immer Cocktails. In den letzten 12 Monaten vor der Befragung hat die Hälfte der Personen, die gemeinsam Drogen auflösen, dazu gebrauchte Spritzen verwendet.

Die häufigsten Techniken des Aufteilens zeigt Abb. 4 (s. auch Tab. 1).

Bei den oben beschriebenen Techniken gelingt es nicht immer, die Drogenlösung „gerecht“ aufzuteilen. Deshalb werden zum Ausgleich verschiedene Vorgehensweisen angewendet: Einen Ausgleich über den Löffel praktizieren $62 \% ; 21 \%$ gleichen über Frontloading und 7\% über Backloading aus.

Die Risikoeinschätzung bei gemeinsamem Auflösen von Drogen zeigt Abb. 5.

$53 \%$ der Konsumenten ziehen das Blut im Verlauf der Drogenapplikation mehrmals auf, dies erfolgt zum Teil zur erneuten Kontrolle der Lage der Nadelspitze, zum Teil aus Gewohnheit. $26 \%$ geben an, nur einmal aufzuziehen. $15 \%$ ziehen kein Blut aktiv auf; sie begnügen sich mit dem spontanen Eintreten von Blut in die Spritze. 


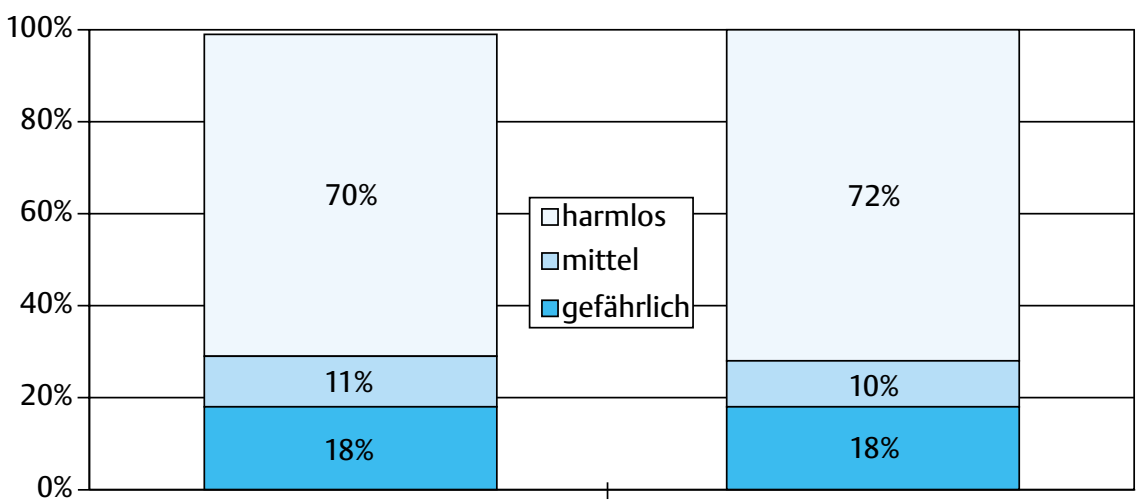

Abb. 5: Einschätzung der Gefährlichkeit der eigenen Technik zum Drogenauflösen $(n=58)$.

\section{Diskussion}

Hepatitis-C-Viren und HIV werden durch Blutübertragung und sexuelle Kontakte übertragen, wobei Letzteres bei Hepatitis $C$ eine marginale Rolle zu spielen scheint [1]. Bei Drogenabhängigen ist das Infektionsrisiko durch Spritzen- und Nadeltausch ein bekanntes Problem und rückte weltweit ins Zentrum von Präventionskampagnen. Dadurch wenig beeinflusst blieb die Ausbreitung von Hepatitis $C$.

Eine Vielzahl von Studien belegt einen Zusammenhang zwischen dem gemeinsamen Zubereiten und Teilen von Drogen zum intravenösen Konsum („indirect sharing“) und viralen Infektionen durch HCV und HIV. Obwohl beim Menschen keine Daten zum Unterschied der Virulenz der beiden Virentypen vorliegen, ist die plausibelste Erklärung, dass für eine Ansteckung mit HCV der Austausch geringerer kontaminierter Blutmengen ausreicht, als dies für HIV-Infektionen notwendig ist. Das „indirect sharing“ muss aufgrund des heutigen Wissensstandes als zentraler Infektionsweg für Hepatitis $C$ bei Drogenabhängigen betrachtet werden. Ein weiterer Hinweis zum Risiko des „indirect sharing“ liefert eine von der ARUD Zürich durchgeführte und ebenfalls in dieser Nummer rezipierte Studie [17]. In gebrauchten Spritzen von HCVpositiven Konsumenten in einem Heroinverschreibungsprogramm ließen sich im Spülwasser mittels PCR beachtliche Viruskonzentrationen nachweisen.

Das Risiko der Übertragung von HIV auf diesem Weg scheint wesentlich kleiner zu sein, doch lässt sich vermutlich ein Teil der neuen HIV-Infektionen, ohne erkennbare andere Risiken, dadurch ebenfalls erklären.

Die vorgestellte Arbeit der ARUD Zürich illustriert die möglichen Wege der Übertragung von Viren beim gemeinsamen Zubereiten von Drogen in Gruppen und die Einschätzung der Infektionsrisiken bei den einzelnen Teilhandlungen. Zusammenfassend können bei folgenden Schritten Blutreste mit infektiösen Partikeln zwischen Konsumierenden ausgetauscht werden:

- Spritzen- und Nadeltausch (nur noch sehr selten und vor allem unter Lebenspartnern praktiziert)

- Mehrfaches Verwenden eigener Spritzen und Nadeln ohne adäquate Reinigung bei

- Teilen der Drogen durch simultanes Aufziehen, Front- oder Backloading

- Zurückführen und Wiederaufziehen der Lösung aus dem Löffel, falls das Teilen nicht „gerecht“ ausgefallen ist.
- Spülen der Spritzen mit Wasser und Aufziehen von Wasser aus einem gemeinsamen Gefäß

- Gemeinsames Verwenden und Austauschen von gebrauchten Filtern

- Gemeinsames Verwenden eines benutzten Löffels

- Gemeinsames Verwenden eines gebrauchten Rührwerkzeugs

Viele Studienteilnehmer sind sich der Risiken nicht bewusst. Auffallend ist, dass das gemeinsame Benutzen von Utensilien als weniger gefährlich betrachtet wird als das Entgegennehmen zum eigenen Gebrauch.

Trotz aller Publikationen zu diesem Thema zeigt sich in unserer klinischen Arbeit und in der Weiterbildungstätigkeit deutlich, dass die Wissenslücken in den vergangen 5 Jahren sowohl bei Fachleuten als auch bei Drogenbenutzern immer noch eklatant sind, so dass die Veröffentlichung unserer Ergebnisse, auch wenn die Daten dazu bereits vor einiger Zeit erhoben wurden, immer noch sinnvoll erscheint.

Zur referierten Studie muss einschränkend gesagt werden, dass es sich bei der Stichprobe um instabile Methadonbezieher handelt. Die Konsumpraktiken sind deshalb nicht repräsentativ für alle i.v. Drogenkonsumenten. In den Gesprächen wurde aber klar, dass ohne Methadonsubstitution dieselben Praktiken häufiger angewandt wurden. Für unbehandelte und randständige Abhängige dürfte sich das Infektionsrisiko beim Drogenkonsum also noch problematischer gestalten.

\section{Resultierende Empfehlungen}

Um Präventionsbotschaften glaubwürdig transportieren zu können, ist es unumgänglich, dass die Beratenden die Praktiken des Injizierens detailliert kennen.

Die Präventionsstrategien sollen sich nicht gegen Konsum in Gruppen richten, weil das gemeinsame, teilweise ritualisierte Konsumieren im Leben der Drogenabhängigen oftmals eine äußerst wichtige Rolle spielt. Dort finden soziale Kontakte statt, die den Austausch von Informationen über Alltagsthemen und Gebiete wie Konsumsicherheit, Stoffreinheit und Gesundheit ermöglichen, was wiederum für Präventionsbemühungen ausgenutzt werden kann. Im Falle einer Überdosierung kann die Gruppe sogar lebensrettend sein. Vielmehr müssen Drogenabhängige über die Gefahren des gemeinsamen Zubereitens von 
Drogen aufgeklärt werden und dabei sichere Injektionspraktiken zum Konsumieren - alleine und in Gruppen - erlernen.

$\mathrm{Zu}$ fordern ist, dass alle notwendigen Utensilien leicht zugänglich und günstig zu erstehen sind. Dies bedeutet unter anderem, das Angebot von Spritzenabgabeprogrammen über Drogeninstitutionen, von Spritzenbussen und von Spritzenautomaten weiter auszudehnen und die Apotheken in das Verteilernetz einzubinden. Saubere Spritzen dürfen von der Polizei nicht mehr konfisziert werden.

Die Befragung hat deutlich gezeigt, dass insbesondere Cocktailund Kokainkonsumenten immer wieder zu exzessivem Konsum neigen, dabei weit mehr Injektionen (bis über 30 pro Tag) applizieren und entsprechend mehr Hilfsmaterial benötigen, als bei ihnen normalerweise verfügbar ist. Die Anzahl der abgegebenen Spritzen sollte deshalb von Abgabestellen nicht limitiert werden. Der Zugang zu Spritzenutensilien darf nicht zu ordnungspolitischen Zwecken missbraucht werden. Es gibt keine Hinweise, dass eine restriktive Abgabe einen in irgendeiner Weise präventiven Charakter hat.

Ein weiteres Gefahrenpotenzial kann durch die breite Abgabe von nur einmal verwendbaren Filtern reduziert werden. Solche Filter speichern wegen ihrer kleinen Membran keine Stoffreste, womit ein wichtiges Motiv für das Aufbewahren wegfällt. Wegen der Porengröße haben aber auch diese Filter nur eine Wirkung auf Schmutzpartikel und Bakterien, nicht aber auf Viren.

\section{Generelle Botschaft zum sicheren intravenösen Konsum}

Für jede Injektion, ob allein oder in Gruppen, gilt:

Eine neue Spritze, einen neuen Filter benutzen, neues NaCl-Wasser direkt aus Einmalgebinde in den Löffel geben. Löffel vor der Drogenzubereitung mit Alkoholtupfer reinigen. Die Injektionsstelle muss vor dem Injizieren desinfiziert werden.

\section{Literatur}

${ }^{1}$ Grob PJ, Negro F, Renner EL im Namen der SEVHEP. Hepatitis C-VirusInfektion. Praxis 2000; 89: 1587-1604

2 Dubois-Arber F, Jeannin A, Spencer B, Meystre-Agustoni G, Haour-Knipe M, Moreau-Gruet F, Benninghoff F, Paccaud F. HIV/AIDS-Prävention in der Schweiz: Bilanz und Ausblick. Schweiz Med Forum 2001; 24: $621-625$
${ }^{3}$ Steffen T, Gutzwiller F. Hepatitis B und C bei intravenös Drogenkonsumierenden in der Schweiz. Praxis 1999; 88: 1937-44

${ }^{4}$ Röhrig S, Grob PJ. Infektionen mit den Hepatitisviren HAV, HBV und HCV sowie mit dem Aidsvirus HIV bei Drogenabhängigen der Gassenszene Zürichs - eine Prävalenzstudie. Schweiz Med Wschr 1990; 120: $621-629$

${ }^{5}$ Somaini B, Wang J, Perozo M, Kuhn F, Meili D, Grob P, Flepp M. A continuing concern: HIV and hepatitis testing and prevalence among drug users in substitution programmes in Zurich, Switzerland. AIDS care 2000; 12 (4): 449-460

${ }^{6}$ Koester S, Booth RE, Zhang Y. The prevalence of additional injectionrelated HIV risk behaviors among injection drug users. J Acquir Immune Defic Syndr Hum Retrovirol 1996; 12 (2): $202-207$

${ }^{7}$ Koester S, Booth R, Wiebel W. The risk of HIV transmission from sharing water, drug mixing containers and cotton filters among intravenous drug users. Intern J Drug Policy 1990; 1 (6): 28 - 30

${ }^{8}$ Grund J, Kaplan C, Adriaans N, Blanken P. Drug sharing and HIV transmission risks: the practice of frontloading in the Dutch injecting drug user population. J Psychoactive Drugs 1991; 23: 1-10

${ }^{9}$ Loimer N, Werner E, Presslich O. Sharing spoons: a risk factor for HIV-1 infection in Vienna. British J Addict 1991; 86: 775 - 778

${ }^{10}$ Jose B, Friedman S, Neaigus A, Curtis R, Grund JP, Goldstein M, Ward T, Des Jarlais DC. Syringe-mediated drug-sharing (backloading): a new risk factor for HIV among injecting drug users. AIDS 1993; 7: $1653-1660$

${ }^{11}$ Stark K, Müller R, Bienzle U, Guggenmoos-Holzmann I. Frontloading: a risk factor for HIV and hepatitis C virus infection among injecting drug users in Berlin. AIDS 1996; 10: 311 - 317

12 Thorpe L, Ouellet L, Hershow R, Bailey S, Williams I, Monerosso E. The multiperson use of non-syringe injection equipment and risk of hepatitis $C$ infection in a cohort of young adult injection drug users, Chicago 1997-1999. Ann epidemiol 2000; 10 (7): $472-473$

${ }^{13}$ Denis B, Dedobbeleer M, Collet T, Petit J, Jamoulle M, Hayani A, Brenard R. High prevalence of hepatitis $C$ virus infection in Belgian intravenous drug users and potential role of the „Cotton-filter“ in transmission: the GEMT study. Acta Gastro-Enterol Belg 2000; 63 (2): 147-53

${ }^{14}$ Hagan H, Thiede H, Weiss NS, Hopkins SG, Duchin JS, Alexander ER. Sharing of drug preparation equipment as a risk factor for hepatitis $C$. Am J Public Health 2000; 91 (1): $42-46$

${ }^{15}$ U.S. Preventive Services Task Force. Guide to clinical preventive services (accessed June 29, 2001, at http://www.ahcpr.gov/clinic/2ndcps/ drugab.pdf). Rockville: Md: Agency for healthcare research and quality 1996; 591

${ }^{16}$ HIV prevention bulletin: medical advice for persons who inject illicit drugs (accessed June 29, 2001, at http://www.cdc.gov/hiv/pubs/ hiv_prev.pdf). Rockville: Md: Public Health Service May 9, 1997

${ }^{17}$ Bertisch-Möllenhoff B, Meili D, Huber M. Nachweis von Hepatitis C-Viruslast in gebrauchten Heroinspritzen. Suchttherapie Supplement 2002; S17-S19

${ }^{18}$ Hagan H, Des Jarlais DC. HIV and HCV infection among injecting drug users. Mount Sinai J Med 2000; $67(5+6): 423-428$

${ }^{19}$ Inciardi JA, Page JB. Drug sharing among intravenous drug users. AIDS 1991; 5 (6): $772-773$ 